\title{
Septic Arthritis of Lumbar Facet Joint with Co-Existing Spondylolisthesis - A Case Report
}

\author{
C Khoo, MBBS (IMU), S Thevarayan, MBBS (RGUHS), P Rengsen, MS Ortho (UM), AS Deepak*, MS Ortho (UM) \\ Department of Orthopaedic \& Traumatology, Malacca General Hospital, Malacca, Malaysia \\ *Prince Court Medical Centre, Kuala Lumpur, Malaysia
}

\begin{abstract}
We report a rare case of septic arthritis of the lumbar facet joint (SALFJ) with co-existing spondylolisthesis of the L5 on S1 vertebra and discuss possible differential diagnoses, diagnostic difficulties and management of this condition.
\end{abstract}

\section{INTRODUCTION}

Septic arthritis of the lumbar facet joint (SALFJ) is a rare clinical entity. Since it was first reported by Halpin in 1981 there have been few other cases reported in literature ${ }^{1}$. The patients are typically older and often immune-compromised. Muffoletto et al, in their review of 27 previously reported cases, concluded that extra-spinal infection and diabetes mellitus are the most common risk factors for developing $\mathrm{SALFJ}^{2}$. However there are reports of SALFJ in younger, immune-competent patients ${ }^{3}$. To our knowledge, SALFJ with co-existing spondylolisthesis of the L5/S1 vertebral level has never been reported. Our purpose is to highlight the diagnostic and management difficulties related to lumbar facet joint infection with spondylolisthesis.

\section{CASE REPORT}

A previously healthy 52 year old Chinese female presented to our outpatient department with progressively worsening back pain of 3 weeks duration. She was seen earlier by another physician for the same complaint 4 days after the initial onset of pain and was managed conservatively with rest and analgesics.

When we saw her, the back pain was so severe she was unable to sit or stand up without assistance. The pain was constant, not relieved by rest and aggravated by the slightest movement of the spine. She did not have preceding fever or any extra-spinal focus of infection. There was no history of acupuncture or injection over the lumbar region. Examination of her spine revealed tenderness over the lower lumbar spine adjacent to the lumbosacral junction involving the paravertebral muscles. She had no neurological deficit in her lower limbs.
Plain radiographs revealed Grade II spondylolisthesis of L5 over S1 with L5 pars defect, decreased L5/S1 disc space and endplate sclerosis and osteophyte formation. As the pain was disproportionate to plain radiograph findings, our initial impression was pyogenic infection of the spine, and an urgent MRI was performed. The MRI showed Meyerding Grade II spondylolisthesis at the L5/S1 vertebral junction with spondylolysis at L5 and disc extrusion at L5/S1 with spinal canal stenosis (Figure $1 \& 2$ ). Blood tests showed elevated infective parameters: total white cell count (TWCC) was high at $15.3 \times 10^{9} / \mathrm{L}$; neutrophilia (88\%); and, erythrocyte sedimentation rate (ESR) was significantly high at $115 \mathrm{~mm} / \mathrm{hr}$. Her urine microscopic examination was suggestive of urinary tract infection and culture grew Escherichia coli sensitive to Cefuroxime. She was started on Cefuroxime based on the urine results. After bed rest for 2 weeks with analgesics and antibiotics, the patient's back pain was better but not fully relieved. Five weeks after initial onset of symptoms, we planned for posterior decompression and fusion for the spondylolisthesis and spinal stenosis to relieve the persistent back pain.

The lumbosacral junction was approached posteriorly and pus was noted under the multifidus muscles on the right side. Debridement of the muscle was performed followed by laminotomy and debridement of the right L5S1 facet joints. Multiple samples were taken and sent for culture. Cultures from the right facet joint, muscle and pus grew methicillin sensitive Staph aureus. Samples taken from the vertebral body and disc space resulted in negative cultures.

Postoperatively and after results of cultures were known, antibiotics were changed from intravenous Cefuroxime to Cloxacillin and Fusidic Acid. The patient was discharged after completing 2 weeks of intravenous the two antibiotics and was given a further course of oral antibiotics for 4 weeks. A lumbar corset was prescribed for 3 months. On follow-up her back pain was improved and the patient showed declining infective parameters; she was pain free on her last follow-up at 1 year following surgical intervention. 


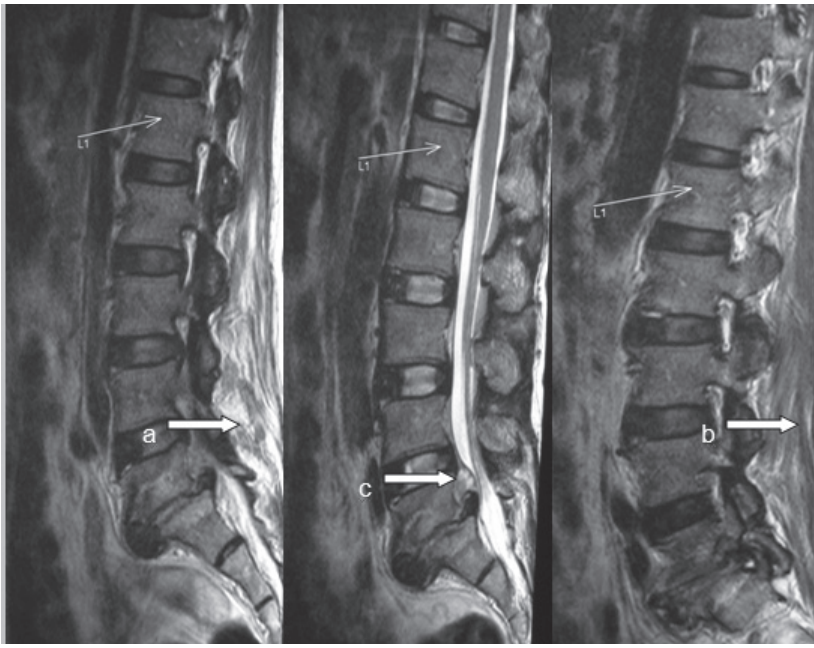

Fig. 1: T2 Weighted Sagittal Images From Right to Left. Right paravertebral muscle show increase signal intensity on $\mathrm{T} 2$ (arrow a) compared to left (arrow b).

Note the degenerative changes in the end plates of $L 5 / S 1$, spondylolisthesis and extruded disc (arrow c) in the central image.

\section{DISCUSSION}

SALFJ is a rare clinical entity. A Medline search for reports of lumbar facet joint septic arthritis with concomitant spondylolisthesis did not yield any results. The usual presentation of lumbar facet joint septic arthritis is acute onset of low back pain, which does not abate with bed rest ${ }^{2,4}$. Compared to spondylodiscitis, SALFJ symptoms tend to be unilateral, present more acutely and with greater severity ${ }^{1,3,4}$. The average duration of symptoms before diagnosis of SALFJ is approximately 4 weeks compared to 3 months for spondylodiscitis ${ }^{2,4}$. The usual infecting organism is Staphylococcus aureus although other organisms have been reported ${ }^{1-5}$.

The patient described in this report had many confounding factors that made early diagnosis difficult. Spondylolisthesis on plain radiograph was a plausible explanation for her back pain; further, her back pain was more diffuse and not unilateral like most reports. In this case, the patient was younger than most other reported cases and she was immune-competent. Although TWCC and ESR were elevated, these parameters could be attributed to urinary tract infection that was found in this patient. Inflammatory markers such as ESR and C-reactive protein are usually elevated in most cases but are non-specific and do not discriminate between SALFJ and spondylodiscitis or other infections ${ }^{1-5}$.

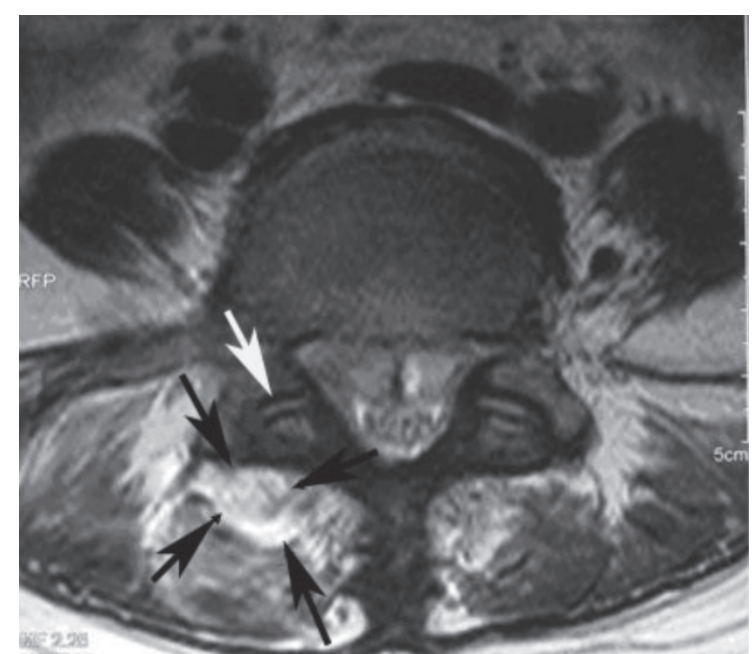

Fig. 2: T2 Weighted Axial View of The L5/S1 Facet Joint And Surrounding Paravertebral Muscle showing erosion of the right L5/S1 facet joint (white arrow) with hyperintense signal in right paravertebral muscles. Note the appearance of a collection around the right L5/S1 joint (black arrows).

Although we initially sought to rule out pyogenic infection of the spine, we were misled by the MRI findings, which suggest spondylolisthesis as the cause of her back pain. On retrospective review of the MRI we found a hyperintense area on $\mathrm{T} 2$ weighted images in the right paravertebral muscles (Figure 1 and 2), which may suggest an underlying inflammatory process or infection. The right L5/S1 facet joint did not show gross erosion or widening of the joint space typical of SALFJ ${ }^{2,4}$. Some authors believe that SALFJ may spontaneously discharge into the paravertebral muscles 1-5. MRI examination with contrast is the imaging modality of choice for diagnosing SALFJ, and clearly demonstrates the presence of facet joint erosion, swelling and paraspinal muscle oedema and abscess ${ }^{2,4}$. Muffoletto et al found that MRI changes consistent with facet joint infection can be demonstrated as early as two days after symptom onset ${ }^{2}$.

SALFJ can be successfully managed conservatively with antibiotic therapy without surgical drainage and debridement ${ }^{1-4}$. Surgical intervention is indicated when neurologic dysfunction is present or when there is persistence of back pain and sepsis despite appropriate antibiotic therapy ${ }^{4}$. In the present case, we did not see further slippage of the L5 on S1 vertebra despite the spondylolisthesis. 


\section{REFERENCES}

1. Halpin DS, Gibson RD. Septic Arthritis of A Lumbar Fact Joint. J Bone Joint Surg. 1987; 69-B: 457-9.

2. Muffoletto AJ, Ketonen LM, Mader JT et al. Hematogenous Pyogenic Facet Joint Infection. Spine. 2001; 26: 1570-6.

3. Rombauts PA, Linden PM, Buyse AJ et al. Septic Arthritis Of A Lumbar Facet Joint Caused By Staphylococcus Aureus. Spine. 2000; 25: 1736-8.

4. Doita M, Nabeshima Y, Nishida K et al. Septic Arthritis Of Lumbar Facet Joints Without Predisposing Infection. J Spinal Discord Tech. 2007; 20: 290-5.

5. Ogura T, Mikami Y, Hase H et al. Septic Arthritis Of A Lumbar Facet Joint Associated With Epidural And Paraspinal Abscess. Orthopedics. 2005; 28: 173-5. 